Note

\title{
Impact of beef extract used for sample concentration on the detection of Escherichia coli DNA in water samples via qPCR
}

\author{
Bernardino Machado-Moreira ${ }^{\mathrm{a}, \mathrm{b}}$, Silvia Monteiro ${ }^{\mathrm{c}}$, Ricardo Santos ${ }^{\mathrm{c}}$, Antonio Martinez-Murcia ${ }^{\mathrm{d}}$, \\ Andreja Rajkovic ${ }^{\mathrm{e}, \mathrm{f}}$, Nada Smigic ${ }^{\mathrm{e}}$, Karl G. Richards ${ }^{g}$, Florence Abram ${ }^{\mathrm{b}}$, Catherine M. Burgess ${ }^{\mathrm{a}, *}$ \\ ${ }^{a}$ Teagasc Food Research Centre, Ashtown, Dublin, Ireland \\ ${ }^{\mathrm{b}}$ Functional Environmental Microbiology, National University of Ireland Galway, Galway, Ireland \\ ${ }^{\mathrm{c}}$ Laboratório de Análises, Instituto Superior Técnico, Universidade de Lisboa, Lisbon, Portugal \\ ${ }^{\mathrm{d}}$ University Miguel Hernandez and Genetic PCR Solutions ${ }^{\mathrm{TM}}$, Alicante, Spain \\ ${ }^{\mathrm{e}}$ Department of Food Safety and Food Quality Management, University of Belgrade-Faculty of Agriculture, Serbia \\ ${ }^{\mathrm{f}}$ Department of Food Technology, Safety and Health, Faculty of Bioscience Engineering, Ghent University; Belgium \\ ${ }^{\mathrm{g}}$ Teagasc Johnstown Castle Environmental Research Centre, Wexford, Ireland
}

\section{A B S T R A C T}

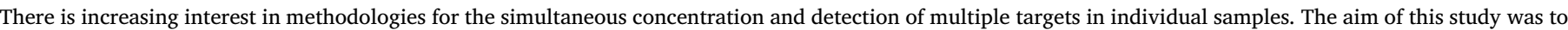

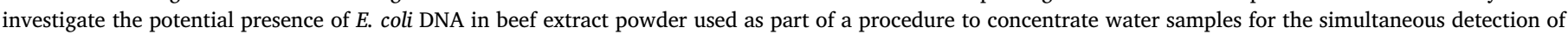

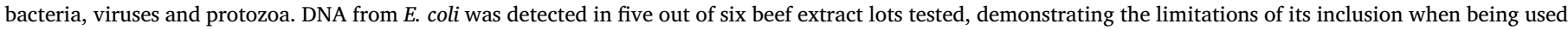

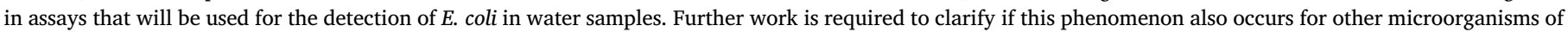
interest in water.

Water used in the production and preparation of vegetables can be sourced from public supply systems, groundwater, recovered rain water, surface water from lakes, rivers or artificial ponds, or even treated wastewater in some countries (Allende and Monaghan, 2015; Jongman and Korsten, 2018; Uyttendaele et al., 2015). The microbial quality of different water sources, especially surface and treated wastewaters is of crucial importance, given the potential for pathogenic microorganisms present in the water to persist and be transferred to plant material. Several fresh produce related microbial disease outbreaks have been attributed to the use of contaminated water at different steps of the farm-to-fork chain, as exemplified by an outbreak in the United States caused by the consumption of raspberries contaminated with Cyclospora present in the water used for pesticide application (Herwaldt et al., 1997), an outbreak in Finland caused by the consumption of frozen raspberries contaminated with Norovirus during irrigation or water spraying before cooling (Ponka et al., 1999), an outbreak attributed to the consumption of spinach leaves contaminated with Escherichia coli O157, in the United States, caused by the use of water contaminated by cattle (Parker et al., 2012; Sharapov et al., 2016), or an outbreak of Salmonella Saintpaul infections in the United States related with the consumption of jalapeno or serrano peppers contaminated with farm water (Barton Behravesh et al., 2011). Ensuring the microbiological quality of the water used in the production of fresh crops is therefore of vital importance for both consumers and food business operators.

Guidelines and regulations for the microbiological quality of water used for the production of crops focus on indicator microorganisms, typically referring to the presence and/or quantification of $E$. coli and faecal or total coliforms as criteria for microbial quality of the irrigation water (Uyttendaele et al., 2015). In Europe, the quality of water used for crop production is regulated by Regulation (EC) 852/2004 on the hygiene of foodstuffs, stating that food business operators producing or harvesting plant products should use potable water or clean water whenever necessary to prevent contamination (European Commission, 2004). The definition of potable water is outlined in EC Regulation 98/ 83/EC on the quality of water intended for human consumption, that states that water that is in contact with food should be of good microbiological quality (European Commission, 1998), with the criteria being established as the absence of $E$. coli and enterococci in $100 \mathrm{~mL}$ of water. The methods specified for the analysis of these two parameters are presented in ISO 9308-1 and ISO 7899-2, respectively. Both standards outline culture methods based on membrane filtration of the water samples, providing results in up to $48 \mathrm{~h}$ after sample processing. Other methodologies, such as the detection of target microorganisms via PCR, have been developed and implemented for several matrices, with the advantages of more rapid results and testing of multiple

\footnotetext{
* Corresponding author at: Teagasc Food Research Centre, Ashtown, Dublin 15, Ireland.

E-mail address: Kaye.burgess@teagasc.ie (C.M. Burgess).
} 
microorganisms from the same sample. A method has been developed to allow for the analysis of large volumes of water for the presence of different microorganisms (Gunnarsdottir et al., 2020). Briefly, a large volume of water undergoes two concentrations steps: primary concentration via dead-end ultrafiltration, and secondary concentration via precipitation of the eluate with PEG and beef extract followed by ultracentrifugation. After sample concentration, DNA and/or RNA are extracted, allowing for the detection of multiple pathogens via qPCR. The primary concentration step is based on particle size exclusion, using a hollow fibre dead-end ultrafiltration setup. This filtration technique has been used as a cost effective alternative for the recovery of different bacteria, virus and protozoa from large water samples (Cashdollar and Wymer, 2013; Hunter et al., 2011; Smith and Hill, 2009). Precipitation of microorganisms from the eluate obtained after the filtration with PEG and beef extract has been documented previously (Lambertini et al., 2008). PEG precipitation is based on the reduction of the solubility of proteins in solution, explained by the excluded volume mechanism (Ingham, 1984). This mechanism is particularly used for the precipitation of viruses from solution. The viral particles in the eluate bind to beef extract, aiding their precipitation (Hill et al., 2005; Hurst et al., 1984; Lewis and Metcalf, 1988; Schwab et al., 1995). The combination of these concentration steps allows for simultaneous detection of bacteria, viruses and protozoa from water samples, providing time and consumable savings (Gunnarsdottir et al., 2020).

A method validation using irrigation and wash water samples collected from different vegetable producers in a number of European countries indicated a high degree of discordance with culture based assays for E. coli. Analysis of internal process controls (i.e. negative controls using sterile distilled water) showed that the utilisation of beef extract during the secondary concentration procedure could result in the detection of $E$. coli DNA in water samples obtained from fresh crop production sites (unpublished data). In order to investigate this, and the associated potential of this technique to generate false positive test results, this study focused on the impact of the utilisation of beef extract during the secondary concentration on the detection of $E$. coli DNA. The secondary concentration step was carried out with mock samples of sterile distilled water (SDW), precipitated with PEG with or without beef extract, followed by the analysis of the precipitates for the presence of $E$. coli DNA.

Samples of beef extract were obtained from different producers in different countries. A total of six samples were collected from five different suppliers across the EU (samples 1 to 6). For each sample, $4 \mathrm{~g}$ of beef extract and $100 \mathrm{~mL}$ of $5 \times$ PEG 8000 (Sigma Aldrich, Ireland) and $\mathrm{NaCl}$ (Sigma Aldrich, Ireland) solution were added to $300 \mathrm{~mL}$ of sterile distilled water (SDW). The mixture was allowed to precipitate overnight at $4{ }^{\circ} \mathrm{C}$. It was then centrifuged at $10,000 \mathrm{~g}$ at $4{ }^{\circ} \mathrm{C}$ for $30 \mathrm{~min}$. The supernatant was discarded, and the precipitate was washed and resuspended in Phosphate Buffered Saline (PBS - Oxoid - ThermoFisher Scientific, Ireland) with $0.001 \%$ Antifoam A (Sigma Aldrich, Ireland) and $0.01 \%$ Tween 80 (Sigma Aldrich, Ireland). DNA from $1 \mathrm{~mL}$ of each sample was extracted with a QIAGEN DNA mini kit (Qiagen, Hilden, Germany), according to the manufacturer's instructions, and preserved at $-80{ }^{\circ} \mathrm{C}$ for later use. A control sample with no added beef extract was processed as described above.

The presence of $E$. coli DNA in each sample was analysed via qPCR, using four different $E$. coli targets. Detection of target sequences was performed using a Roche 480 LightCycler II (Roche, Penzberg, Germany). For the detection of the uidA gene, the primers uidAF ( 5'-CAACGAACTGAACTGGCAG-3') and uidAR (5'-CATTACGCTGCGAT GGAT-3') were used (Abberton et al., 2016; Chern et al., 2009). The $\operatorname{rodA}$ gene was detected using the primers rodA984F (5'- GCAAACCA CCTTTGGT-3') and rodA984R (5'- CTGTGGGTGTGGATTGACAT-3') (Abberton et al., 2016). The tuf gene was detected using the primers TEcol553F (5'- TGGGAAGCGAAAATCCTG-3') and TEcol754R (5'CAGTACAGGTAGACTTCTG-3') (Abberton et al., 2016). qPCR reactions
Table 1

Detection of E. coli DNA in sterile distilled water precipitated with PEG and beef extract from different suppliers. Results are presented as the number of amplification cycles $(\mathrm{Ct})$ required for detection of target DNA. Confirmatory gel electrophoresis results are presented in brackets (positive/negative). The SYBR Green qPCR protocol was run with 45 repetitions of the amplification cycle, and the GPS ${ }^{\mathrm{TM}}$ qPCR kit protocol was run with 40 repetitions of the amplification cycle. Late amplification results (in the last five amplification cycles of each kit protocol) are presented as $>40.00$ or $>35.00$ due to the probability of nonspecific target amplification, leading to a higher uncertainty in the result.

\begin{tabular}{llllll}
\hline Sample & Supplier & \multicolumn{2}{l}{ SYBR Green qPCR Ct } & \multirow{2}{*}{$\begin{array}{l}\text { GPS EscCol } \\
\text { qPCR kit Ct }\end{array}$} \\
\cline { 3 - 5 } & & uidA & rodA & tuf & \\
\hline 1 & 1 & $27.05(+)$ & $30.21(+)$ & $34.81(+)$ & $27.49(+)$ \\
2 & 1 & $35.52(+)$ & $39.47(+)$ & $>40.00(+)$ & $>35.00(+)$ \\
3 & 2 & $32.21(+)$ & $34.51(+)$ & $35.52(+)$ & $31.99(+)$ \\
4 & 3 & $35.59(+)$ & $>40.00(-)$ & $>40.00(+)$ & $>35.00(+)$ \\
5 & 4 & $25.59(+)$ & $28.65(+)$ & $29.22(+)$ & $27.75(+)$ \\
6 & 5 & nd (-) & nd (-) & nd $(-)$ & nd $(-)$ \\
\hline
\end{tabular}

were performed using the Lightcycler 480 SYBR Green I Master Kit (Roche, Penzberg, Germany), following the qPCR kit manufacturer's instructions: one initial activation step of $5 \mathrm{~min}$ at $95{ }^{\circ} \mathrm{C}$, followed by 45 cycles of $10 \mathrm{~s}$ of denaturation at $95^{\circ} \mathrm{C}, 10 \mathrm{~s}$ of annealing at $60{ }^{\circ} \mathrm{C}$ and $10 \mathrm{~s}$ of elongation at $72{ }^{\circ} \mathrm{C}$. The GPS ${ }^{\mathrm{TM}}$ EscCol dtec-qPCR kit $\left(\mathrm{GPS}^{\mathrm{TM}}\right.$, Alicante, Spain) was also used for detection of $E$. coli DNA in the tested samples. The primers and probe used in the assay were undisclosed. qPCR reactions were performed according to the manufacturer's instructions: one initial activation step of $15 \mathrm{~min}$ at $95{ }^{\circ} \mathrm{C}$, followed by 40 cycles of a $15 \mathrm{~s}$ denaturation step at $95{ }^{\circ} \mathrm{C}$ and an extension step of $60 \mathrm{~s}$ at $60{ }^{\circ} \mathrm{C}$. The results were recorded as amplification threshold crossing point values $(\mathrm{Ct})$ of each $\mathrm{qPCR}$ reaction. For confirmatory purposes, the qPCR products of each reaction were run on agarose gels for DNA visualisation. The qPCR products obtained from the reactions targeting the uidA gene were sequenced at Eurofins Genomics (Ebersberg, Germany) using the primer uidAF. The obtained sequences were compared to the non-redundant database of sequences at the National Center for Biotechnology Information (NCBI), using BLASTN (basic local alignment search tool) algorithm (Zhang et al., 2000).

E. coli DNA was detected in five out of the six tested samples (Table 1). In samples BE1-BE5, the qPCR reactions were positive for the uidA, $\operatorname{rod} A$ and tuf genes, and the five samples also tested positive for $E$. coli DNA using the GPS ${ }^{\mathrm{TM}}$ EscCol dtec-qPCR kit. All qPCR positive results were confirmed by gel electrophoresis, with the exception of the $\operatorname{rodA}$ gene fragment amplified from sample BE4, whose qPCR result corresponded to a later amplification signal, suggesting a lower target DNA concentration (Table 1). Further confirmation of the presence of $E$. coli DNA in samples $1-5$ was obtained by sequencing the DNA fragments obtained in the reaction targeting the uidA gene (Table 2). No E. coli DNA was detected in sample 6, in any of the tested qPCR reactions. Additionally, qPCR reactions for the control sample were negative for all tested targets.

\section{Table 2}

Phylogenetic affiliations of the gene fragments obtained from the qPCR reaction targeting the uidA gene of $E$. coli. DNA sequences were compared to those available from the non-redundant database of sequences at the National Center for Biotechnology Information (NCBI) as of the 26th of August of 2019, using BLASTN (basic local alignment search tool) algorithm.

\begin{tabular}{lll}
\hline Sample & Closest match (accession no.) & Similarity (\%) \\
\hline 1 & E. coli (CP042934) & 98.78 \\
2 & E. coli (CP019213) & 94.03 \\
3 & E. coli (CP042953) & 97.94 \\
4 & E. coli (CP019213) & 98.72 \\
5 & E. coli (CP019213) & 98.67
\end{tabular}


Beef extract is generally prepared by dehydration of beef tissue infusions, obtaining a paste or dried powder. It is used as a nutritive ingredient in different microbiological culture media, as an undefined source of amino acids, nitrogen, vitamins and carbon (Cote, 2009; Vanderzant and Splittstroesser, 2001). Beef extract has also been extensively used in the elution and precipitation of viruses from filters and water samples (Lambertini et al., 2008; Hill et al., 2005; Hurst et al., 1984; Lewis and Metcalf, 1988; Schwab et al., 1995; Ikner et al., 2012; Rhodes et al., 2016; Staggemeier et al., 2015). Other products used for the same purpose, such as meat peptones, are usually obtained from animal protein sources such as muscle, bone or skin tissues, which are hydrolysed with enzymes isolated from animal sources, including pepsin or trypsin. In order to preserve nutrients lost during the protein hydrolysis treatments used in production of these products, beef extract is not submitted to harsh hydrolysis treatments $\left(\mathrm{BBL}^{\mathrm{TM}}\right.$ Beef Extract Powder, Bacto ${ }^{\mathrm{TM}}$ Beef Extract, Desiccated, n.d). If animal gut tissues are used for the production of beef extract, $E$. coli cells present in the tissue may persist during the manufacturing process, leading to the presence of this strain or its DNA in the final product.

The presence of $E$. coli DNA in beef extract which is used in a method designed to concentrate water samples for detection of different microorganisms raises important limitations. Given the importance of E. coli as an indicator microorganism used to monitor water quality, the utilisation of the proposed method for the detection of this species may be of limited utility, due to the potential of the technique to generate false positive results. In a food and water testing context, false positive results can lead to economic and reputational damage to food business operators, due to possible rejection of products or product recalls. It is therefore imperative to ensure that the method of choice is capable of detecting the microorganisms of interest with high sensitivity, avoiding false positive results. Alternatives based on centrifugation and membrane filtration for the secondary concentration of water samples are available (Gunnarsdottir et al., 2020), but those are designed specifically for detection of bacteria. Nonetheless, the method evaluated in this study remains a useful alternative for simultaneous detection of other pathogenic bacteria, viruses and protozoa from water samples, and further research on a potential alternative for replacement of beef extract for viral precipitation would improve the applicability of the developed method.

This work was supported by the EU project AQUAVALENS FP7KBBE-311846 and the Teagasc Walsh Fellowship no. 2015034.

\section{Author statement}

Machedo-Moreira: conceptualization, methodology, validation, investigation, writing-original draft.

Monteiro: resources, writing-review and editing.

Santos: conceptualization, writing-review and editing.

Martinez-Murcia: resources, writing-review and editing.

Rajkovic: conceptualization, writing-review and editing.

Smigic: resources, writing-review and editing.

Richards: supervision, writing-review and editing.

Abram: supervision, writing-review and editing.

Burgess: conceptualization, supervision, writing-review and editing.

\section{Declaration of Competing Interest}

The authors declare that they have no known competing financial interests or personal relationships that could have appeared to influence the work reported in this paper.

\section{References}

Abberton, C.L., et al., 2016. Survival, biofilm formation, and growth potential of environmental and enteric Escherichia coli strains in drinking water microcosms. Appl. Environ. Microbiol. 82 (17), 5320-5331.

Allende, A., Monaghan, J., 2015. Irrigation water quality for leafy crops: a perspective of risks and potential solutions. Int. J. Environ. Res. Public Health 12 (7), 7457-7477.

Barton Behravesh, C., et al., 2011. 2008 Outbreak of Salmonella Saintpaul infections associated with raw produce. N. Eng. J. Med. 364 (10), 918-927.

BBL $^{\mathrm{TM}}$ Beef Extract Powder, Bacto ${ }^{\mathrm{TM}}$ Beef Extract, Desiccated, [cited 2019 26/08/2019]; Available from: https://www.bdbiosciences.com/documents/Beef_Extract.pdf.

Cashdollar, J.L., Wymer, L., 2013. Methods for primary concentration of viruses from water samples: a review and meta-analysis of recent studies. J. Appl. Microbiol. 115 (1), 1-11.

Chern, E.C., et al., 2009. Comparison of fecal indicator bacteria densities in marine recreational waters by QPCR. water quality. Expo. Health 1 (3), 203-214.

European Commission, 1998. Council Directive 98/83/EC of 3 November 1998 on the Quality of the Water Intended for Human Consumption.

European Commission, 2004. Regulation (EC) No 852/2004 of the European Parliament and of the Council of 29 April 2004.

Cote, R.J., 2009. Media composition, microbial, laboratory scale. Encyclopedia of industrial biotechnology: bioprocess, bioseparation, and cell. Technol. 1-20.

Gunnarsdottir, M.J., et al., 2020. Water safety plan enhancements with improved drinking water quality detection techniques. Sci. Total Environ. 698, 134185.

Herwaldt, B.L., et al., 1997. An outbreak in 1996 of cyclosporiasis associated with imported raspberries. N. Engl. J. Med. 336 (22), 1548-1556.

Hill, V.R., et al., 2005. Development of a rapid method for simultaneous recovery of diverse microbes in drinking water by ultrafiltration with sodium polyphosphate and surfactants. Appl. Environ. Microbiol. 71 (11), 6878-6884.

Hunter, D.M., et al., 2011. Dead-end ultrafiltration concentration and IMS/ATP-bioluminescence detection of Escherichia coli O157:H7 in recreational water and produce wash. J. Microbiol. Methods 87 (3), 338-342.

Hurst, C.J., et al., 1984. Comparison of commercial beef extracts and similar materials for recovering viruses from environmental samples. Can. J. Microbiol. 30 (10), 1253-1263.

Ikner, L.A., Gerba, C.P., Bright, K.R., 2012. Concentration and recovery of viruses from water: a comprehensive review. Food Environ. Virol. 4 (2), 41-67.

Ingham, K.C., 1984. [20] Protein precipitation with polyethylene glycol. In: Methods In Enzymology. Academic Press, pp. 351-356.

Jongman, M., Korsten, L., 2018. Irrigation water quality and microbial safety of leafy greens in different vegetable production systems: a review. Food Rev. Int. 34 (4), 308-328.

Lambertini, E., et al., 2008. Concentration of enteroviruses, adenoviruses, and noroviruses from drinking water by use of glass wool filters. Appl. Environ. Microbiol. 74 (10), 2990-2996.

Lewis, G.D., Metcalf, T.G., 1988. Polyethylene-glycol precipitation for recovery of pathogenic viruses, including hepatitis-a virus and human rotavirus, from oyster, water, and sediment samples. Appl. Environ. Microbiol. 54 (8), 1983-1988.

Parker, C.T., et al., 2012. Distinct transcriptional profiles and phenotypes exhibited by Escherichia coli O157:H7 isolates related to the 2006 spinach-associated outbreak. Appl. Environ. Microbiol. 78 (2), 455-463.

Ponka, A., et al., 1999. An outbreak of calicivirus associated with consumption of frozen raspberries. Epidemiol. Infect. 123 (3), 469-474.

Rhodes, E.R., et al., 2016. The evaluation of hollow-fiber ultrafiltration and celite concentration of enteroviruses, adenoviruses and bacteriophage from different water matrices. J. Virol. Methods 228, 31-38.

Schwab, K.J., De Leon, R., Sobsey, M.D., 1995. Concentration and purification of beef extract mock eluates from water samples for the detection of enteroviruses, hepatitis a virus, and Norwalk virus by reverse transcription-PCR. Appl. Environ. Microbiol. 61 (2), 531-537.

Sharapov, U.M., et al., 2016. Multistate Outbreak of Escherichia coli O157:H7 Infections Associated with Consumption of Fresh Spinach: United States, 2006. J. Food Prot. 79 (12), 2024-2030.

Smith, C.M., Hill, V.R., 2009. Dead-end hollow-Fiber ultrafiltration for recovery of diverse microbes from water. Appl. Environ. Microbiol. 75 (16), 5284-5289.

Staggemeier, R., et al., 2015. Molecular detection of human adenovirus in sediment using a direct detection method compared to the classical polyethylene glycol precipitation. J. Virol. Methods 213, 65-67.

Uyttendaele, M., et al., 2015. Microbial hazards in irrigation water: standards, norms, and testing to manage use of water in fresh produce primary production. Compr. Rev. Food Sci. Food Saf. 14 (4), 336-356.

Vanderzant, C., Splittstroesser, D., 2001. Compendium of Methods for the Microbiological Examination of Foods. American Public Health Association, Washington, DC, USA.

Zhang, Z., et al., 2000. A greedy algorithm for aligning DNA sequences. J. Comput. Biol. 7 (1-2), 203-214. 